\title{
Composição quali-quantitativa da produção de 'Syrah' cultivada sob estresse hídrico transiente
}

\author{
Antonio O. Santos ${ }^{1} \&$ Oren Kaye ${ }^{2}$
}

\begin{abstract}
RESUMO
A produção de vinho de qualidade requer o cultivo de uvas de padrão superior, com características químicas e físicas favoráveis, resultantes das condições micrometeorológicas locais, da variedade e do manejo do solo e da cultura. A composição quantitativa e qualitativa do rendimento da videira pode ser responsiva à variação na lâmina de irrigação e ao sistema de poda utilizado. Doravante, a interação entre os dois fatores citados e o meio físico circundante foi mostrada ser influente na qualidade do vinho. Um experimento foi conduzido com uva vinífera nas estações de crescimento de 2003 a 2005; cujo objetivo foi determinar a melhor combinação entre sistemas de poda (manual e mecânica) e manejo da água, para a produção de vinho de 'Syrah' (Vitis vinifera L.) cultivada em condições de microclima quente e seco. Os resultados mostraram que a composição do rendimento foi consistentemente afetada pelo sistema de poda, enquanto o melhor perfil aromático foi observado para 'Syrah', cultivada sob poda mecânica e estresse hídrico baixo a moderado.
\end{abstract}

Palavras-chave: Vitis vinífera L., microclima, irrigação

\section{Composition and chemical-sensorial profile of 'Syrah' cultivated under transient water stress}

\begin{abstract}
The production of a quality wine depends on the cultivation of high quality grapes with favorable chemical and physical characteristics, which result from the submitted micrometeorological regime, variety and soil-plant management. Grape yield and quality performance can be responsive to varying levels of water depth and the pruning system. The interaction between these two factors and the surrounding environment has been proved to be influential upon the wine quality. An experiment was carried out with wine grapes through the growing seasons of 2003 to 2005 . The objective was to determine the best combination of pruning (manual and mechanized) and irrigation practices on 'Syrah' (Vitis vinifera L.) envisaging wine quality achievement, under a hot and dry microclimate. Results showed that the grape composition was consistently affected by the pruning system whereas the best aroma profile was observed in mechanically pruned plots cultivated under low to moderate water stress.
\end{abstract}

Key words: Vitis vinífera L., microclimate, irrigation 


\section{INTRODUÇÃO}

A videira possui mecanismos fisiológicos de auto-regulação, direcionando suas reservas para vigor (crescimento vegetativo) ou frutificação (crescimento reprodutivo), de acordo com suas próprias necessidades (K eller et al., 2008). Geralmente se objetiva, em vitivinicultura, adotar estratégias de manejo para direcionar os recursos da planta visando a qualidade da produção, tal como acúmulo de precursores de fenóis, o que permitirá maior complexidade de sabor e aroma, além de maior expressão varietal (Deloire et al., 2002). Tipicamente, um vinho superior requer bagas de uva que tenham razão casca/suco alta, pois a maior parte dos fenóis extraíveis está na casca. A razão citada depende de se ter cachos com bagas de menor tamanho (Gurovich \& Vergara, 2005); por outro lado, a produtividade deve ser levada em consideração pois, atualmente, a uva é vendida por peso e não por qualidade. Este aparente paradoxo entre qualidade e produtividade tem levado a investigações sobre um ponto de equilíbrio para a otimização da qualidade, sem penalizar significativamente a produtividade (carga de frutos) (Poni et al., 1994; Ferreyra et al., 2004). Notadamente, este equilíbrio tem sido buscado pelo rigor em práticas de manejo e, em particular, pelo manejo da videira em relação à disponibilidade da água no solo e pelo ajuste da carga de frutos.

Sob níveis não restritivos de água no solo, o crescimento vegetativo é excessivo e compete com as bagas por assimilados. 0 dossel pode, doravante, fechar, tendo efeitos negativos sobre a iniciação das gemais florais, maturação dos frutos e fitossanidade (Dokoozlian \& Kliewer, 1996); por outro lado, um déficit hídrico muito severo pode causar redução de assimilados e afetar negativamente a produtividade e a qualidade da uva, notadamente pela limitação fotossintética e/ou excessiva exposição dos cachos à radiação solar (Teixeira et al., 2002).

A diminuição do crescimento vegetativo é o resultado mais imediato da diminuição da água no solo (H siao, 1973), sendo mais sensitivo ao déficit hídrico do que as trocas gasosas. Tal fato foi demonstrado experimentalmente em videiras viníferas (Bindi et al., 2005; Bindon et al., 2008). Quanto a esta questão, vários trabalhos têm demonstrado que a irrigação deficitária controlada (RDI), ou estresse hídrico transiente, pode ser uma forma de balancear entre carga de frutos e vegetação, adotando-se a recarga hídrica no solo somente quando os níveis da água no solo caem abaixo de certo patamar crítico previamente determinado (Soar \& Loveys, 2007).

Outra estratégia crítica de manejo da videira é a poda mecanizada. Este tipo de poda é menos precisa e, tipicamente, sua implementação resulta em uma carga maior de gemas por planta (quando comparada com a poda manual); geralmente, leva ao redor de três anos para o vinhedo se aclimatar total mente à mudança de poda manual para a poda mecânica (Freeman \& K liewer, 1983). 0 custo de produção e a disponibilidade de mão-de-obra são alguns dos fatores determinantes do uso da poda mecânica, onde as condições topográficas permitem sua implementação.

A pesar da realização de uma série de estudos, ainda não se conseguiu elucidar totalmente a relação básica entre estresse hídrico, carga de frutos e qualidade do vinho. A maioria deles mostra que níveis ótimos de disponibilidade hídrica no solo atrasam a acumulação de sólidos solúveis e incrementa o tamanho da baga (Freeman et al., 1980, K asimatis, 1977), ambos deletérios à qualidade do vinho. Contrariamente, noutros trabalhos e sob as mesmas condições, a maturação foi acelerada (Hardie \& Considine, 1976). 0 aumento da lâmina de água reduziu a qualidade do vinho quando a produtividade de parreiras irrigadas foi limitada por poda severa; no entanto, o abaixamento da qualidade do vinho sob ótimas condições de água no solo foi associado ao aumento no tamanho da baga (Freeman et al., 1980). Zyl \& Weber (1977) demonstraram que a disponibilidade hídrica otimizada atrasou a maturação da baga, mas outros parâmetros de qualidade do fruto não foram afetados.

Conforme relatado, pairam ainda dúvidas sobre a interação mais benéfica entre sistema de poda (carga de frutos) e recarga hídrica, para os mais diferentes regimes microclimáticos.

Objetivou-se, com este estudo, determinar a influência da prática das podas manual e mecânica e de estresse hídrico transiente sobre a qualidade da uva e perfil aromático do vinho da cultivar Syrah (Vitis vinífera L.), durante três safras agrícolas.

\section{MATERIAL E MÉTODOS}

\section{Local, época e cultivar}

0 experimento foi conduzido na região sul de F resno, no vale de San Joaquin (CA, EUA ), em uma área de 4,5 ha. 0 clima é caracterizado por verões quentes e secos com persistência de altos valores de déficit de pressão de vapor durante a estação de crescimento e desenvolvimento da videira. A maior parte da chuva se concentra no inverno. 0 clima se enquadra como BWk na classificação de Koeppen. No sistema de classificação climática californiana, situa-se na região V (Winkler et al., 1974), o que significa tratar-se da região com mais al to número possível de graus-dia, na estação de crescimento.

O solo da área apresentava textura uniforme, de média a arenosa (sandy loam).

0 vinhedo foi estabelecido em 1998 pela enxertia a campo de 'Syrah' ( $V$. vinifera) sobre porta-enxertos Harmony (Solanis $\mathrm{x}$ Dogridge $\mathrm{x}$ Othello) pre-estabelecidos.

0 sistema de condução constou de cordão bilateral, com um só fio para sustentação dos ramos (catch wire). A s linhas de videiras estavam orientadas no sentido leste-oeste, com espaçamento de 3,3 $\mathrm{m}$ entre fileiras e 2,4 $\mathrm{m}$ entre plantas.

\section{Delineamento estatístico}

Dois tratamentos de poda foram aplicados no experimento: a) poda manual (HP) aplicada na última metade do período de dormência da videira, mantendo-se de 40 a 60 gemas por planta, em cordão esporonado, e b) poda mecânica (MP) do tipo "over the row" - com lâminas de corte horizontal, dispostas em cilindros verticais e lâminas frontais de 
corte vertical" $\left(90^{\circ}\right.$ vertical e $0^{\circ}$ horizontal), constituindose em uma área de ataque ("box cut") de 0,45 x 0,45 m sobre o cordão. Um podador hidráulico-mecânico de acoplamento frontal foi utilizado em conjunto com um trator (Polimate, A ustrália).

Três lâminas de recarga hídrica foram aplicadas no experimento. Os tratamentos de irrigação consistiram na suspensão do fornecimento da recarga, a partir do estabelecimento do fruto (meados de maio) até que o potencial da água na folha atingisse, ao redor do meio-dia, -1,1,-1,3 e $-1,5 \mathrm{M} \mathrm{pa}$, constituindo-se níveis de estresse hídrico baixo, médio e severo, respectivamente.

Da combinação entre os dois sistemas de poda e os três níveis de estresse hídrico transientes, seis tratamentos foram estabel ecidos e cada um repetido cinco vezes, totalizando 30 linhas com 160 videiras para cada linha.

0 arranjo experimental consistiu de um delineamento em blocos casualizados, com seis tratamentos e cinco repetições, provenientes da combinação entre três lâminas de recarga hídrica e dois sistemas de poda (mecânica e manual).

\section{Dados micrometeorológicos e água no solo}

A irrigação nas linhas individuais foi implementada através de microgotejamento, com três gotejadores por planta. A recarga hídrica em cada nível de estresse foi implementada pela combinação da observação de sensores de monitoramento do conteúdo da água no solo, estimativas da evapotranspiração, potencial da água na folha e desenvolvimento do dossel.

Em junho de 2003 vinte e oito poços de observação (tubos de acesso) foram instalados no perfil do solo da área experimental, para observação do conteúdo da água no solo. 0 monitoramento foi feito semanalmente, utilizando-se um sensor móvel, baseado na capacitância dielétrica e acoplado a um microdatalloger (Prism System, Irrigation Scheduling $M$ ethods Inc.). 0 sensor móvel determinava a umidade do solo pela interpretação da condutância dielétrica nos perfis dos poços de observação $(30,60$ e $90 \mathrm{~cm})$.

0 potencial da água na folha foi monitorado semanalmente em folhas não sombreadas, localizadas na parte externa do dossel, utilizando-se uma câmara de pressão do tipo SchoIlander (PMD Industries). Duas folhas totalmente expandidas foram tomadas aleatoriamente, em cada repetição, ao redor do meio-dia; depois de envolvidas com saco plástico fino, as folhas foram seccionadas da planta e as leituras de potencial tomadas imediatamente.

A evapotranspiração da cultura (ETC) foi determinada segundo metodologia descrita para videira (Williams \& M athews, 1990).

Quando necessário, todas as variáveis meteorológicas foram tomadas a partir da estação meteorológica automática da localidade de "Five Points", pertencente à rede de estações automáticas CIM IS (California Irrigation M anagement Information System) e distante $5 \mathrm{~km}$ do experimento.

\section{Composição do rendimento}

Seis videiras foram amostradas, al eatoriamente, em cada tratamento, perfazendo 180 videiras no total determinando- se, por ocasião da colheita, o peso por baga, o número de bagas por cacho, o peso do cacho, o número de cachos por planta e o rendimento por planta e por área.

\section{Vinificação}

Para microvinificação, as repetições foram agrupadas e elaborados seis estilos de vinho a partir dos seis tratamentos, procurando-se representar os procedimentos industriais usuais da região de estudo.

A proximadamente $240 \mathrm{~kg}$ de uva foram usados para vinificação em cada tratamento; após a colheita, os lotes de uva foram transportados para o laboratório e imediatamente vinificadas.

As uvas foram desengaçadas sem se proceder ao esmagamento; após o desengace, as uvas foram colocadas em contêineres de polietileno (grau alimento) de capacidade para $500 \mathrm{~L}$; a seguir, fez-se a adição de $\mathrm{SO}_{2}$ na forma de metabissulfito de potássio, deixando-se o mosto em pré-maceração durante $24 \mathrm{~h}$, a $18^{\circ} \mathrm{C}$; decorrido este tempo o mosto foi inoculado com leveduras secas ativas (Saccharomyces cerevisiae); em seguida, os contêineres foram colocados em sala com temperatura controlada a $25^{\circ} \mathrm{C}$ e três remontas foram feitas durante 0 dia; a fermentação foi monitorada para verificação do açúcar residual e desprendimento do $\mathrm{CO}_{2}$, através de densímetro e termômetro.

A pós a "secagem" o mosto foi transferido para uma prensa hidrostática de duas tonel adas de capacidade (Vaslin-B ucher, França); aplicou-se uma pressão constante de $0,8 \mathrm{~B}$ ar; a prensa foi rotacionada e re-pressurizada duas vezes para cada lote de vinho; feita a prensagem os vinhos foram postos em contêineres de $47 \mathrm{~L}$.

Com o propósito de estabilidade e garantia de que todos os lotes de vinho estivessem sujeitos à mesma condição, eles foram inoculados com viniflora Oenos (Fugelsang, 1996), para indução da fermentação malolática, a temperatura de $20^{\circ} \mathrm{C}$; durante esta fase os contêineres foram periodicamente tratados com nitrogênio expansivo, para eliminar eventual presença de oxigênio e evitar a formação de $\mathrm{H}_{2} \mathrm{~S}$ (gás sulfídrico), fazendo-se as transfegas pertinentes ao processo, de acordo com o padrão industrial usual (Zoecklein et al., 1999). A fermentação malolática foi monitorada através de espectroscopia de infravermelho (Kupina \& Shrinkhande, 2003), após o que se mantiveram os vinhos fabricados por aproximadamente quinze dias, a temperatura de $-3^{\circ} \mathrm{C}$ para estabilização; fez-se o controle do nível de $\mathrm{SO}_{2}$ (A storia Pacific Flow) e os vinhos foram engarrafados e mantidos a temperatura de $18{ }^{\circ} \mathrm{C}$.

\section{Análise química da uva, mosto e vinho}

0 conteúdo de fenóis totais, teor de sólidos solúveis, pH, acidez titulável, potássio e intensidade de cor, foram determinados através de varredura em subamostras, utilizando-se espectroscopia de infravermelho (Winescan, Foss Eletric, Dinamarca). Para os anos de 2004 e 2005 uma análise do conteúdo de antocianina na baga colhida foi efetuada, conforme Iland et al. (2004); para isto, a polpa da casca de amostras de uva foi removida a mão, enxaguada com água destilada e secada em papel toal ha; as cascas foram pesadas e colocadas em tubos 
apropriados para centrifugação, contendo $50 \mathrm{~mL}$ de metanol acidificado ( $\left.1 \% \mathrm{HCl} \mathrm{V} \mathrm{v}^{-1}\right)$; a seguir, os tubos foram armazenados 48 h no escuro; continuando, após diluição apropriada com metanol acidificado, a absorbância de uma alíquota de $5 \mathrm{~mL}$ do extrato foi tomada em espectrofotômetro na microbanda de $520 \mathrm{~nm}$ (Spectronic, Rochester NY). A concentração de antocianina ( $\mathrm{mg} \mathrm{g}^{-1}$ de casca) foi determinada usandose os valores do peso molecular (529) e a absorbância molar (28.000) para glicosideo-3-malvidina.

\section{Análise do perfil aromático do vinho}

Estudaram-se os efeitos dos sistemas de poda e estresses hídricos transientes sobre o perfil aromático do vinho; para isto, 28 painelistas previamente treinados compuseram 0 corpo de julgamento para os vinhos; através do teste do triângulo (Lawless \& Heymann, 1998) detectaram-se diferenças sensoriais individuais nos tratamentos; as diferenças encontradas foram descritas quantitativamente, implementando-se uma análise descritiva, com base no julgamento da intensidade de percepção (escala não estruturada) dos principais descritores para o vinho de 'Syrah' da região do experimento. Utilizou-se o "círculo aromático" (Noble et al., 1987; Duncan, 1999), do qual se derivaram os componentes enquadrados na faixa de variação de aromas, descrito na literatura para o vinho de 'Syrah'; cada um dos vinhos (de cada tratamento) foi submetido a cada um dos painelistas.

Os dados da análise sensorial foram submetidos à análise de variância e as diferenças significativas foram detal hadas pelo teste de Tukey.

\section{RESULTADOS E DISCUSSÃO}

A Figura 1 representa a evolução da temperatura média do ar, na área do experimento. Os dados revelam uma região na qual a maturação da uva ocorre com temperaturas médias superiores a $16{ }^{\circ} \mathrm{C}$ e os dados mostram, sobretudo, que as temperaturas máximas do ar se aproximam de $40^{\circ} \mathrm{C}$ no "veraison". De acordo com Jackson \& Lombard (1993), a evolução da temperatura, tal como descrito para a área, se enquadra na faixa de região "B eta", já que a época de maturação da uva coincide com temperaturas médias do ar superiores a $16{ }^{\circ} \mathrm{C}$. D oravante, a região tem, marcadamente, a persistência de alto déficit de pressão de vapor ao longo da estação vegetativa, com acentuação no ciclo reprodutivo. Este padrão, que compõe um al to valor de demanda evaporativa atmosférica, está de acordo com o regime climático da região, notadamente um clima de deserto com a chuva concentrada no inverno. Se, por um lado, se nota uma diferenciação razoável entre temperatura média do ar diurna e noturna (benéfica à síntese dos precur-

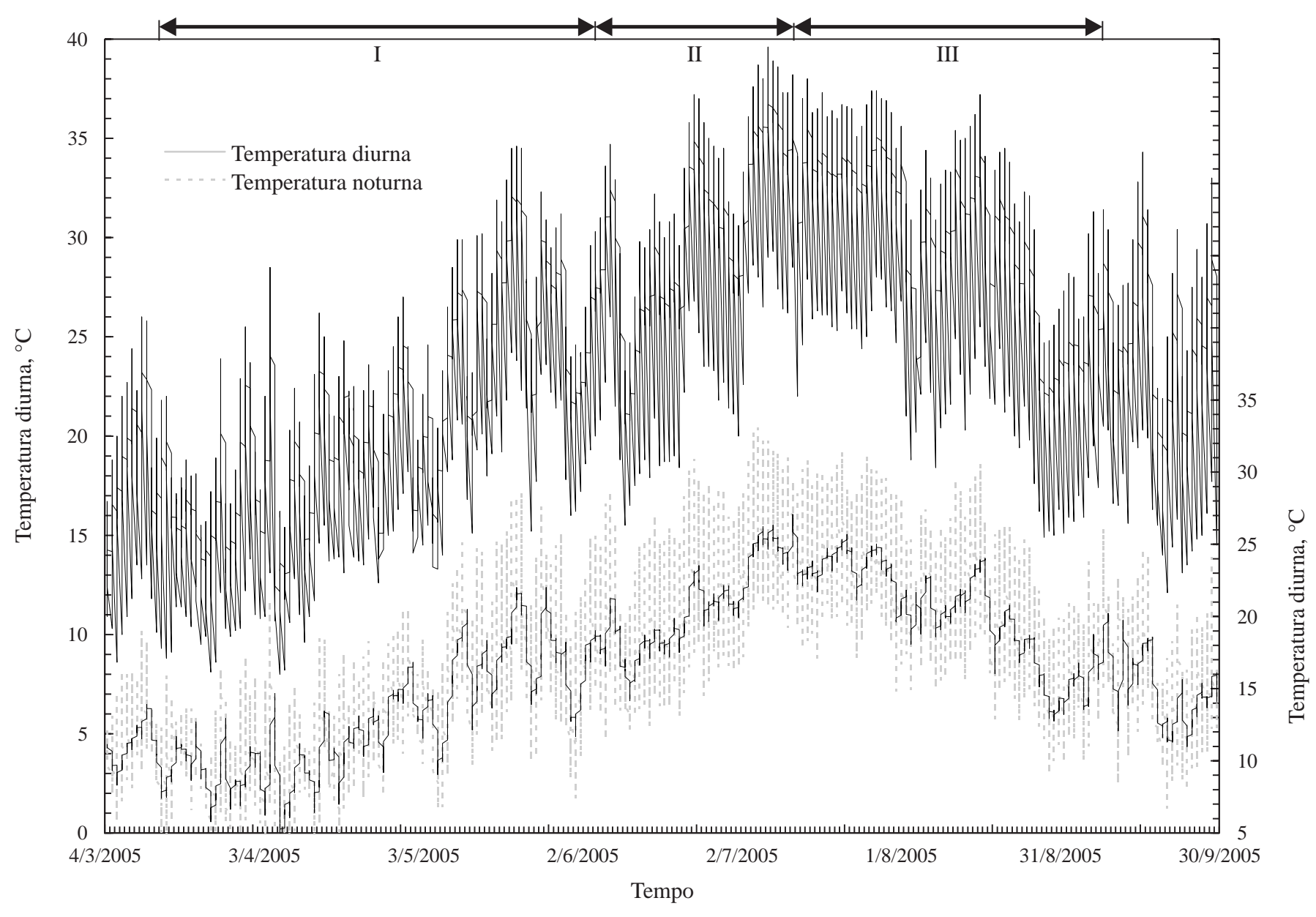

Figura 1. Evolução da média horária móvel para a temperatura do ar, diurna e noturna, próximo da área experimental, durante a estação de crescimento de 2005. As fases fenológicas são mostradas: I - Abertura de gemas- estabelecimento do fruto; II- Estabelecimento do fruto - veraison; III - Veraison - colheita 
sores de aroma e sabor), por outro se evidencia a incidência de temperaturas elevadas, alcançando a fase III da formação da baga, o que pode prejudicar a qualidade da uva (J ensen et al., 2007; Tarara et al., 2008).

$\mathrm{Na}$ comparação entre os anos do experimento e se considerando a lâmina d'água total aplicada no experimento e a evapotranspiração da cultura (Tabela 1), evidencia-se que 0 estresse hídrico foi intensificado ao longo dos anos, particularmente para as parcelas conduzidas sob estresse hídrico severo; pode-se observar, na Tabela 2, que este fenômeno não resultou em tendência de decréscimo na produtividade da cultivar ao longo do tempo. O correu uma flutuação da produtividade cuja melhor performance foi obtida no último ano do experimento, apesar do aumento da pressão do estresse hídrico (Tabela 1), fato que mostra a grande resistência desta cultivar ao estresse hídrico e seu grande poder de adaptação, mesmo quando cultivada sob pressão de extremos microclimáticos, quando os efeitos do estresse hídrico são intensificados.

Tabela 1. Razão entre recarga hídrica e consumo hídrico (ETc) ao longo de três estações de crescimento e desenvolvimento da cultivar Syrah

\begin{tabular}{lccc}
\hline \multirow{2}{*}{ Água aplicada/Uso da água } & \multicolumn{3}{c}{ Razão anual } \\
\cline { 2 - 4 } Recarga hídrica sob estresse hídrico baixo/ETc & $\mathbf{2 0 0 3}$ & $\mathbf{2 0 0 4}$ & $\mathbf{2 0 0 5}$ \\
Recarga hídrica sob estresse hídrico moderado/ETc & $\mathbf{0 , 6 2}$ & 0,67 & 0,56 \\
Recarga hídrica sob estresse hídrico severo/ETc & - & 0,54 & 0,46 \\
\hline
\end{tabular}

$\mathrm{Na}$ Tabela 2 se acham os resultados obtidos na análise dos componentes do rendimento da produção da cultivar Syrah, nos três anos consecutivos de observação. Comparando-se 0 sistema de poda mecânica (M P) com o sistema de poda manual (HP), observam-se diferenças significativas em vários itens dos componentes do rendimento, as quais se mantiveram ao longo dos anos do experimento. Sob o sistema de poda mecânica as bagas produzidas são mais leves e em menor número no cacho; além disso, os cachos são em maior número, mais leves e, portanto, menores. Devido ao maior número de cachos por planta, resultantes da poda mecânica, a produção final por planta e a produção por área foram maiores neste sistema de poda. Nota-se que a aplicação do estresse hídrico controlado resultou em maior área superficial específica de casca, em função do maior número de bagas de menor peso; tal fato tem importância para a extractibilidade de polifenóis retidos na casca por ocasião da maceração. A produção por área, para os dois sistemas de poda, foi significativamente diferente em todos os anos do experimento (Tabela 2) mostrando que, se por um lado os valores por hectare não têm grande amplitude (HP XMP), por outro não houve compensação entre os sistemas, de modo a se assemel harem produtividades nos três anos do experimento.

A nalisando-se individualmente os níveis de estresse hídrico aplicados, nota-se na Tabela 2 que a disponibilidade hídrica influenciou a produção por planta e a produção por área em todos os anos do experimento; do mesmo modo se constata que 0 estresse hídrico transiente influenciou o peso

Tabela 2. Composição do rendimento da cultivar Syrah, conduzida em dois sistemas de poda e três lâminas de irrigação, em três safras consecutivas, em Fresno, no Vale Central do Estado da Califórnia (EUA)

\begin{tabular}{|c|c|c|c|c|c|c|c|c|c|c|c|c|}
\hline & \multicolumn{4}{|c|}{2003} & \multicolumn{4}{|c|}{2004} & \multicolumn{4}{|c|}{2005} \\
\hline & & Bx & Mod & Sev & & Bx & Mod & Sev & & Bx & Mod & Sev \\
\hline \multicolumn{13}{|c|}{ Produtividade $\left(\mathrm{t} \mathrm{ha}^{-1}\right)$} \\
\hline & & $26,23 \mathrm{a}$ & $23,64 \mathrm{~b}$ & $21,03 \mathrm{c}$ & & $19,2 \mathrm{a}$ & $13,2 \mathrm{~b}$ & $11,6 \mathrm{~b}$ & & $30,57 \mathrm{a}$ & $28,21 \mathrm{~b}$ & $26,34 \mathrm{c}$ \\
\hline $\mathrm{HP}$ & $20,52 \mathrm{a}$ & $21,96 \mathrm{a}$ & $20,63 a b$ & $18,88 \mathrm{~b}$ & $13,2 \mathrm{a}$ & $17,5 \mathrm{a}$ & $12,0 \mathrm{~b}$ & $10,3 \mathrm{~b}$ & 26,74 a & $29,73 \mathrm{a}$ & 26,16 a & $24,33 \mathrm{a}$ \\
\hline MP & $26,79 \mathrm{~b}$ & $30,25 \mathrm{c}$ & $26,67 \mathrm{~d}$ & $23,20 \mathrm{e}$ & $16,1 \mathrm{~b}$ & $20,8 \mathrm{~d}$ & $14,6 \mathrm{e}$ & $12,9 \mathrm{e}$ & $30,00 \mathrm{~b}$ & $31,41 \mathrm{a}$ & $30,25 \mathrm{a}$ & $28,35 \mathrm{a}$ \\
\hline \multicolumn{13}{|c|}{ Rendimento por planta $(\mathrm{kg})$} \\
\hline & & 20,78 a & $18,73 \mathrm{~b}$ & $16,66 \mathrm{c}$ & & $15,2 \mathrm{a}$ & $10,5 b$ & $9,2 \mathrm{~b}$ & & $24,22 \mathrm{a}$ & $22,35 \mathrm{~b}$ & $20,87 \mathrm{c}$ \\
\hline $\mathrm{HP}$ & $16,23 \mathrm{a}$ & $17,39 \mathrm{a}$ & $16,34 a b$ & $14,96 \mathrm{~b}$ & $10,5 \mathrm{a}$ & $13,9 \mathrm{a}$ & $9,5 \mathrm{a}$ & $8,2 \mathrm{a}$ & $21,19 \mathrm{a}$ & $23,55 \mathrm{a}$ & $20,73 \mathrm{a}$ & $19,28 \mathrm{a}$ \\
\hline MP & $21,22 \mathrm{~b}$ & $24,18 \mathrm{c}$ & $21,13 d$ & $18,37 \mathrm{e}$ & $12,8 \mathrm{~b}$ & $16,5 \mathrm{a}$ & $11,6 \mathrm{a}$ & $10,2 \mathrm{a}$ & $23,77 \mathrm{~b}$ & $24,88 \mathrm{a}$ & $23,97 \mathrm{a}$ & $22,46 \mathrm{a}$ \\
\hline \multicolumn{13}{|c|}{ Peso de cacho (g) } \\
\hline & & $152,8 \mathrm{ab}$ & $150,9 \mathrm{~b}$ & $136,4 \mathrm{c}$ & & $147,0 \mathrm{a}$ & 95,2 b & $91,5 b$ & & $117,62 \mathrm{a}$ & $118,85 \mathrm{a}$ & $110,34 \mathrm{~b}$ \\
\hline $\mathrm{HP}$ & $172,73 a$ & $176,5 \mathrm{a}$ & $179,4 \mathrm{a}$ & $162,3 \mathrm{a}$ & $140,1 \mathrm{a}$ & $183,3 \mathrm{a}$ & $117,6 \mathrm{a}$ & $119,3 \mathrm{a}$ & $148,90 \mathrm{a}$ & $149,34 \mathrm{a}$ & $154,24 \mathrm{a}$ & $143,11 \mathrm{a}$ \\
\hline MP & $120,72 b$ & $129,1 \mathrm{a}$ & $122,4 \mathrm{a}$ & $110,6 \mathrm{a}$ & $82,4 \mathrm{~b}$ & $110,7 \mathrm{a}$ & $72,8 \mathrm{a}$ & $63,7 \mathrm{a}$ & $82,04 \mathrm{~b}$ & $85,90 \mathrm{a}$ & 83,46 a & $77,58 \mathrm{a}$ \\
\hline \multicolumn{13}{|c|}{ No de cachos por planta } \\
\hline & & 145,4 a & $136,5 a b$ & $133,6 \mathrm{~b}$ & & $129,2 \mathrm{a}$ & $124,1 \mathrm{a}$ & $125,8 \mathrm{a}$ & & $227,29 a$ & $214,22 \mathrm{a}$ & $215,42 \mathrm{a}$ \\
\hline $\mathrm{HP}$ & $97,1 \mathrm{a}$ & $103,0 \mathrm{a}$ & $94,4 \mathrm{a}$ & 93,8 a & $82,1 \mathrm{a}$ & $85,0 \mathrm{a}$ & $80,1 \mathrm{a}$ & $81,3 \mathrm{a}$ & $144,07 \mathrm{a}$ & $159,40 \mathrm{a}$ & $136,55 \mathrm{a}$ & $136,25 \mathrm{a}$ \\
\hline MP & $179,9 \mathrm{~b}$ & $187,9 \mathrm{a}$ & $178,5 \mathrm{a}$ & $173,4 \mathrm{a}$ & $170,5 \mathrm{~b}$ & $173,3 \mathrm{a}$ & $168,0 \mathrm{a}$ & $170,3 \mathrm{a}$ & $293,89 \mathrm{~b}$ & $295,19 \mathrm{a}$ & $291,90 \mathrm{a}$ & $294,59 \mathrm{a}$ \\
\hline \multicolumn{13}{|c|}{ No bagas por cacho } \\
\hline & & $186,8 \mathrm{a}$ & $182,1 \mathrm{a}$ & $194,0 \mathrm{a}$ & & $139,6 \mathrm{a}$ & $148,4 \mathrm{a}$ & $136,6 \mathrm{a}$ & & $151,90 \mathrm{a}$ & $152,83 \mathrm{a}$ & $160,67 \mathrm{a}$ \\
\hline $\mathrm{HP}$ & $224,2 \mathrm{a}$ & $217,1 \mathrm{a}$ & 236,3 a & $219,2 \mathrm{a}$ & $164,4 \mathrm{a}$ & $165,0 \mathrm{a}$ & $165,8 \mathrm{a}$ & $162,5 \mathrm{a}$ & 183,08 a & $177,25 \mathrm{a}$ & $182,67 \mathrm{a}$ & $189,33 \mathrm{a}$ \\
\hline MP & $151,1 \mathrm{~b}$ & $156,5 \mathrm{a}$ & $128,0 \mathrm{a}$ & $168,9 \mathrm{a}$ & $118,6 \mathrm{~b}$ & $114,2 \mathrm{a}$ & $131,0 \mathrm{a}$ & $110,7 \mathrm{a}$ & $127,18 \mathrm{~b}$ & $126,56 \mathrm{a}$ & $123,00 \mathrm{a}$ & $132,00 \mathrm{a}$ \\
\hline \multicolumn{13}{|c|}{ Peso de baga $(\mathrm{g})$} \\
\hline & & & & & & $1,47 \mathrm{a}$ & $1,07 \mathrm{~b}$ & $1,02 \mathrm{bc}$ & & $1,27 \mathrm{a}$ & $1,13 b$ & $1,08 \mathrm{~b}$ \\
\hline $\mathrm{HP}$ & & & & & $1,29 \mathrm{a}$ & $1,57 \mathrm{a}$ & $1,17 \mathrm{a}$ & $1,13 \mathrm{ba}$ & $1,21 \mathrm{a}$ & $1,34 \mathrm{a}$ & $1,18 \mathrm{a}$ & $1,12 \mathrm{a}$ \\
\hline MP & & & & & $1,09 \mathrm{~b}$ & $1,38 \mathrm{a}$ & $0,98 \mathrm{a}$ & $0,91 \mathrm{a}$ & $1,10 \mathrm{~b}$ & $1,20 \mathrm{a}$ & $1,08 \mathrm{a}$ & $1,03 \mathrm{a}$ \\
\hline
\end{tabular}

Valores externos, em cada quadrícula, representam a variação em função do estresse hídrico (horizontal) ou do sistema de poda (vertica ). Valores internos, em cada quadrícula, representam eventuais interações entre tipo de poda e nível de estresse hídrico. Valores seguidos da mesma letra (na linha ou coluna) não diferem pelo teste de Tukey em nível de 5\%. Bx, Mod e Sev são os níveis de estresse hídrico baixo, moderado e severo, respectivamente. HP - poda manual; MP - poda mecânica 
de cachos em todas as safras e o número de cachos por planta somente em 2003; no entanto, um nível de estresse hídrico moderado influenciou significativamente o peso da baga nos dois anos observados (2003 e 2004), não se diferenciando significativamente do nível de estresse hídrico severo.

A nalisados conjuntamente o sistema de poda e o estresse hídrico transiente, nota-se que houve interação significativa somente entre os dois fatores na produção final por planta e por área em 2003 e 2004 e na produção por área em 2004 (Tabela 2); portanto, o efeito da poda é mais claro e mais consistente que o efeito do estresse hídrico, considerando-se os três anos analisados, já que o sistema de poda é uma função da carga de gemas remanescentes por planta, enquanto o estresse hídrico é um fenômeno mais complexo, dependente de inúmeras interações no sistema solo-planta-atmosfera.

Os resultados das análises químicas da uva, do mosto e do vinho, ao longo das vindimas, estão na Tabela 3, na qual se tem que os valores de sólidos solúveis na colheita foram influenciados pelo sistema de poda, nos anos de 2003 e 2005, sendo que o estresse hídrico somente influenciou significativamente esta variável no ano de 2004; nota-se, por outro lado, que os teores de sólidos solúveis são al tos para todos os anos, assim como são baixos os valores de acidez total na maioria das safras, o que se deve, em parte, ao regime microclimático local, marcado por altas temperaturas (Tabela 3). Souza et al. (2002) e A morim et al. (2005) obtiveram valores médios de 17 e $21,5^{\circ}$ Brix para 'Syrah' em ciclo reprodutivo estival e outonal, respectivamente, em microclima de altitude no Sudoeste do Brasil. Girona et al., (2006) relataram valores médios de $22,3^{\circ} \mathrm{Brix}$, para a mesma cultivar na Espanha.

Quanto à acidez, valores mais altos em acidez titulável foram relatados para cultivares tintas no Sul do B rasil (Rizzon \& M iele, 2002). Os valores baixos para acidez total, tal como aqueles determinados na safra de 2003 (Tabela 3), sugerem a correção da acidez do vinho, prática contemplada pela atual legislação do vinho na região do presente estudo (Galpin, 2006).
Particularmente, para a safra de 2005 o nível de potássio presente no mosto se diferenciou significativamente entre os níveis de estresse (Figura 2F); tal fato concorda com a diferenciação da acidez total em 2005, que foi menor para as parcelas com maior estresse hídrico, em que a absorção de potássio foi maior.

Os valores de antocianina e fenóis totais, como indicativos mais robustos da qualidade dos vinhos provenientes das três vindimas mostram, de "per se", que os valores são mais baixos do que aqueles observados em 'Syrah' por Ginestar et al. (1998) e D eloire \& H unter (2005), respectivamente na A ustrália e na França.

No ano em que a produtividade foi menor (2004), o efeito do estresse hídrico foi claramente indutor de maior acúmulo nesses parâmetros (Tabela 3); da mesma maneira, o sistema de poda influenciou aquele acúmulo; para o ano de 2004, os valores mostram uma influência bem marcada, tanto da poda como do estresse hídrico transiente; já para 2003 e muito embora não exista o dado sobre antocianina, a Tabela 3 e a Figura 2A indicam que não houve diferença significativa nos valores para fenóis totais nem na intensidade de cor, entre os níveis de estresse hídrico ou sistema de poda; enfim, para o ano de 2005 os valores de fenóis totais foram influenciados significativamente pelo sistema de poda (Tabela 3), mas não houve aparente influência, seja do sistema de poda ou do nível de estresse sobre os níveis de antocianina, naquele ano.

Para todas as safras analisadas o tamanho da baga foi consistentemente influenciado pelo nível de estresse e pelo sistema de poda (Tabela 2) (fato que se deriva da observação do peso e número de baga por cacho); apesar disso, o acúmulo de antocianina e polifenóis somente foi consistentemente influenciado pelo estresse hídrico e sistema de poda em 2004; este fato se confirma através da Figura 2B, na qual se nota que a intensidade de cor foi significativamente influenciada pelo nível de estresse hídrico.

Tabela 3. Resultados das análises químicas feitas na uva, no mosto e no vinho de Syrah durante três safras consecutivas

\begin{tabular}{|c|c|c|c|c|c|c|c|c|c|c|c|c|}
\hline & \multicolumn{4}{|c|}{2003} & \multicolumn{4}{|c|}{2004} & \multicolumn{4}{|c|}{2005} \\
\hline & & Bx & Mod & Sev & & Bx & Mod & Sev & & Bx & Mod & Sev \\
\hline \multicolumn{13}{|c|}{${ }^{\circ}$ Brix - colheita } \\
\hline & & 23,98 a & $25,51 \mathrm{a}$ & $25,25 \mathrm{a}$ & & $23,11 a b$ & $22,96 \mathrm{a}$ & $23,88 \mathrm{~b}$ & & $22,37 \mathrm{a}$ & $22,27 \mathrm{a}$ & $22,85 \mathrm{a}$ \\
\hline $\mathrm{HP}$ & $25,80 \mathrm{a}$ & 25,38 a & $26,29 \mathrm{a}$ & 25,74 a & $23,25 \mathrm{a}$ & $23,14 \mathrm{a}$ & $22,84 \mathrm{a}$ & $23,76 \mathrm{a}$ & $23,37 \mathrm{a}$ & 23,18 a & $23,06 \mathrm{a}$ & $23,86 \mathrm{a}$ \\
\hline MP & $24,02 \mathrm{~b}$ & $22,58 \mathrm{a}$ & $24,72 \mathrm{a}$ & $24,76 \mathrm{a}$ & 23,39 a & $23,08 \mathrm{a}$ & $23,08 \mathrm{a}$ & $24,00 \mathrm{a}$ & $21,63 \mathrm{~b}$ & $21,56 \mathrm{a}$ & $21,48 \mathrm{a}$ & $21,48 \mathrm{a}$ \\
\hline \multicolumn{13}{|c|}{ Acidez total $\left(\mathrm{g} \mathrm{L}^{-1}\right)$ - colheita } \\
\hline & & $3,41 \mathrm{a}$ & $3,31 \mathrm{a}$ & $3,25 \mathrm{a}$ & & $5,8 \mathrm{a}$ & $6,3 \mathrm{a}$ & 6,3 a & & $4,25 \mathrm{a}$ & $4,17 \mathrm{ab}$ & $4,05 \mathrm{~b}$ \\
\hline $\mathrm{HP}$ & $3,43 \mathrm{a}$ & $3,46 \mathrm{a}$ & $3,46 \mathrm{a}$ & $3,37 \mathrm{a}$ & $6,2 \mathrm{a}$ & $6,3 \mathrm{a}$ & $6,1 \mathrm{a}$ & $6,2 \mathrm{a}$ & $4,20 \mathrm{a}$ & $4,63 \mathrm{a}$ & $4,10 \mathrm{~b}$ & $3,88 \mathrm{bc}$ \\
\hline MP & $3,22 b$ & $3,36 \mathrm{a}$ & $3,15 \mathrm{a}$ & $3,14 \mathrm{a}$ & $6,1 \mathrm{a}$ & $5,4 \mathrm{a}$ & $6,4 \mathrm{a}$ & $6,4 \mathrm{a}$ & $4,11 \mathrm{a}$ & $3,88 \mathrm{~d}$ & 4,24 be & 4,21 ef \\
\hline \multicolumn{13}{|c|}{ Antocianina $\left(\mathrm{g} \mathrm{L}^{-1}\right)$ - baga } \\
\hline & & & & & & $0,63 \mathrm{a}$ & $0,71 \mathrm{~b}$ & $0,74 \mathrm{~b}$ & & $0,731 \mathrm{a}$ & $0,724 \mathrm{a}$ & $0,694 \mathrm{a}$ \\
\hline $\mathrm{HP}$ & & & & & $0,66 \mathrm{a}$ & $0,63 \mathrm{a}$ & $0,67 \mathrm{a}$ & $0,68 \mathrm{a}$ & $0,727 \mathrm{a}$ & $0,764 \mathrm{a}$ & $0,725 \mathrm{a}$ & $0,693 \mathrm{a}$ \\
\hline MP & & & & & $0,72 \mathrm{~b}$ & $0,62 \mathrm{a}$ & $0,75 \mathrm{a}$ & $0,80 \mathrm{a}$ & $0,706 \mathrm{a}$ & $0,699 \mathrm{a}$ & $0,723 \mathrm{a}$ & $0,695 \mathrm{a}$ \\
\hline \multicolumn{5}{|c|}{ Fenóis totais - mosto (mg GAE g-1) } & \multicolumn{8}{|c|}{ Fenóis totais - vinho $\left(\mathrm{mg} \mathrm{GAE} \mathrm{g} \mathrm{g}^{-1}\right)$} \\
\hline & & 13,69 a & $16,16 \mathrm{a}$ & 17,04 a & & $30,02 \mathrm{a}$ & $34,61 \mathrm{~b}$ & $37,27 \mathrm{c}$ & & $31,61 \mathrm{a}$ & $34,63 \mathrm{a}$ & 34,39 a \\
\hline HP & $17,22 \mathrm{a}$ & $15,97 \mathrm{a}$ & $15,04 \mathrm{a}$ & $18,66 \mathrm{a}$ & 35,16 a & $30,93 \mathrm{a}$ & 36,58 a & $37,99 \mathrm{a}$ & $31,82 \mathrm{a}$ & $31,39 \mathrm{a}$ & $32,59 \mathrm{a}$ & $31,48 \mathrm{a}$ \\
\hline MP & $14,02 \mathrm{a}$ & $11,41 \mathrm{a}$ & $15,27 \mathrm{a}$ & $15,37 \mathrm{a}$ & $32,76 \mathrm{~b}$ & $29,10 \mathrm{a}$ & $32,64 \mathrm{a}$ & $36,55 \mathrm{a}$ & $35,27 \mathrm{~b}$ & $31,84 \mathrm{a}$ & $36,68 \mathrm{~b}$ & $37,31 \mathrm{~b}$ \\
\hline
\end{tabular}

Valores externos, em cada quadrícula, representam a variação em função do estresse hídrico (horizontal) ou do sistema de poda (vertica ). Valores internos, em cada quadrícula, representam eventuais interações entre tipo de poda e nível de estresse hídrico. Valores seguidos da mesma letra (na linha ou coluna) não diferem pelo teste de Tukey em nível de 5\%. Bx, Mod e Sev são os níveis de estresse hídrico baixo, moderado e severo, respectivamente. HP - poda manual; MP - poda mecânica 


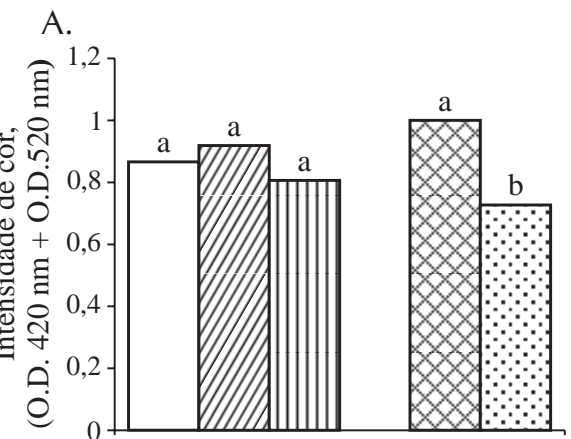

B.
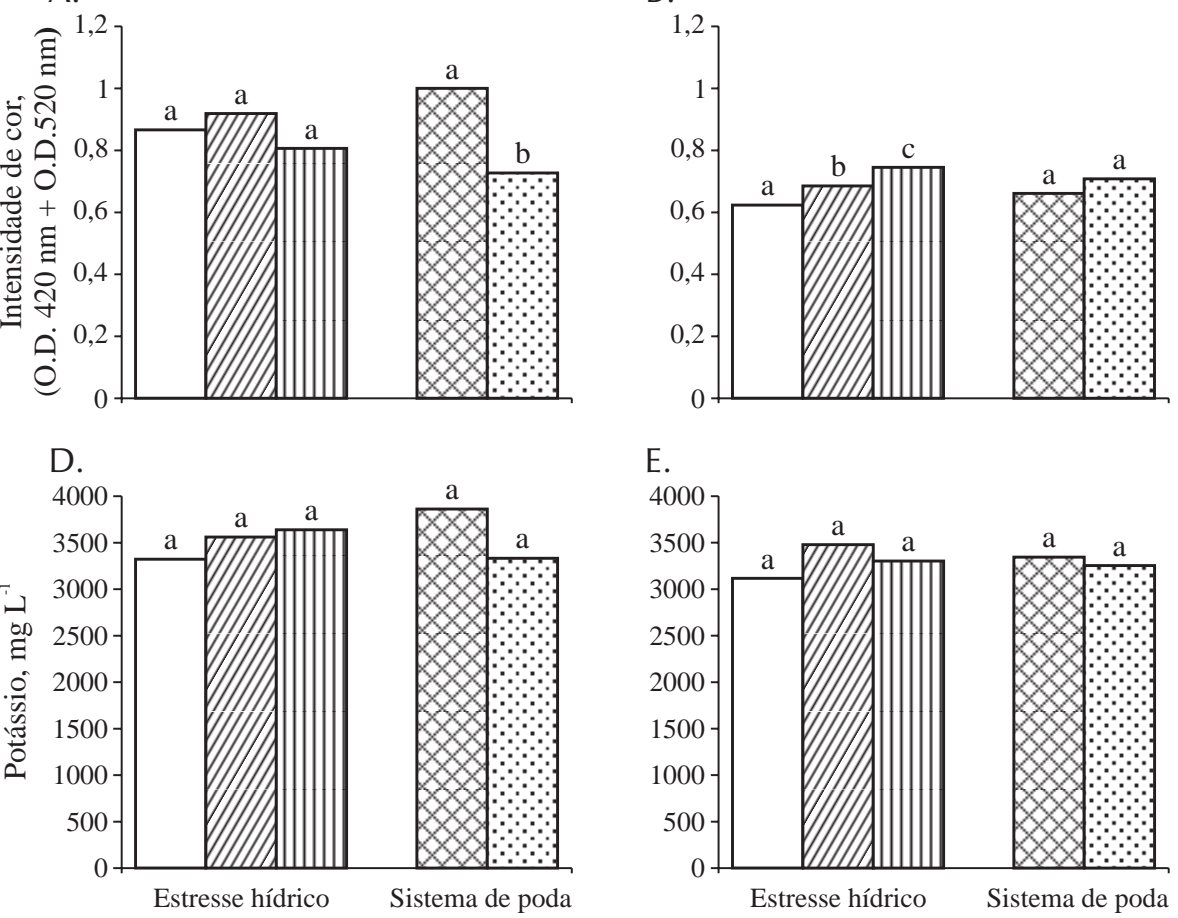

E.

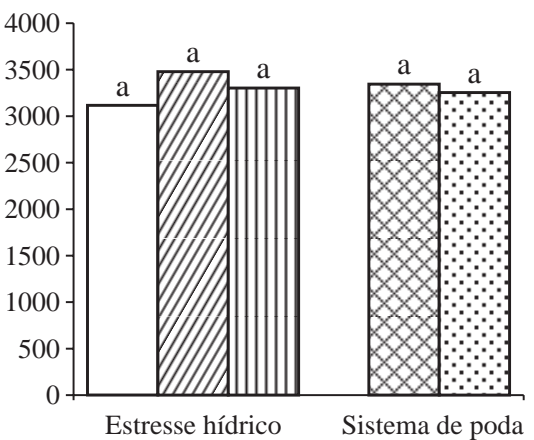

C.

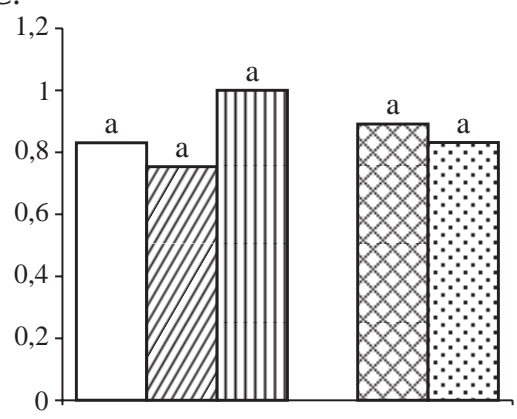

F.

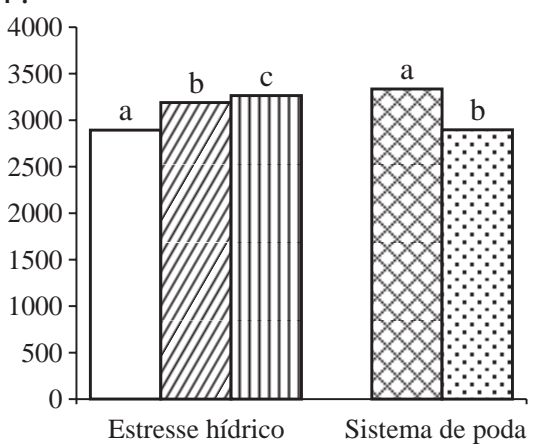

Obs.: letras iguais não diferem ao nível de 5\% pelo teste de Tukey. Inexiste interação entre sistema de poda e nível de estresse hídrico

$\begin{array}{lll}\square \text { Baixo estresse hídrico } & \text { Poda manual } \\ \text { DZ Estresse hídrico moderado } & \\ \square \text { Estresse hídrico severo } & \end{array}$

Figura 2. Intensidade de cor e teor de potássio no mosto de Syrah, durante as safras de 2003 (A, D), 2004 (B, E) e 2005 (C, F)

Freeman \& K liewer (1983), B ravdo et al. (1985) relataram aumento em antocianina e fenóis totais, portanto, na qualidade do vinho, devido à redução da lâmina de irrigação em 'Cabernet Franc', 'Syrah', 'C abernet Sauvignon' e 'Carignane', respectivamente. Neste trabalho, apenas na safra de 2004 se deu uma clara relação entre aumento da qualidade da uva através do aumento do nível de estresse e pela variação do sistema de poda; sugere-se, para 0 ano de 2005, que 0 aumento da intensidade do estresse (Tabela 1) ocasionou uma exposição maior do cacho nas parcelas mais estressadas, o que foi observado no experimento, sobretudo após o "veraison"; a escaldadura do cacho nas parcelas mais estressadas pode ter influenciado a biossíntese de polifenóis e antocianina, levando a um nivelamento entre os ganhos em qualidade advindos da suspensão da irrigação e/ou variação no sistema de poda e as perdas por extremos de incidência da radiação solar; para 2003, aquela exposição foi ainda mais grave, haja vista que o nível de estresse severo havia sido estabelecido inicialmente em -1,6 M pa, o que causou desfoIhamento intenso.

Em relação a este aspecto, Dokoozlian (2001) observou, em 'C abernet Sauvignon', que uma exposição excessiva dos cachos à radiação direta el evou a sua temperatura, e indicou indesejáveis reduções no acúmulo de cor, fenóis totais e malato na baga, por ocasião da colheita.

A Tabela 4 mostra os resultados do teste do triângulo efetuado para verificar eventuais diferenças entre os vinhos provenientes dos diversos tratamentos, na safra de 2004; conclui-se que houve diferença significativa entre os níveis de estresse hídrico; os pares de vinho 1, 4 e 6 mostram que os vinhos provenientes de parcelas contrastantes são, quanto à disponibilidade hídrica, diferentes; além disso, o par

Tabela 4. Resultados do painel sensorial para o teste do triângulo (duo/ trio) utilizando-se 27/28 painelistas treinados, para diferenciação dos vinhos de 'Syrah' elaborados no experimento

\begin{tabular}{|c|c|c|c|}
\hline \multicolumn{4}{|c|}{ Estresse hídrico dentro do Sistema de Poda } \\
\hline Pares de vinhos - HP & $\begin{array}{l}\text { Respostas } \\
\text { corretas/ } \\
\text { incorretas }\end{array}$ & $\begin{array}{l}\text { Risco de aceitar } \\
\text { um vinho como } \\
\text { diferente, quando } \\
\text { não o é (\%) }\end{array}$ & $\begin{array}{c}\text { Significância } \\
\text { estatística }\end{array}$ \\
\hline 1 - Baixo x Severo & $25 / 27$ & 0,01 & $S^{* \star *}$ \\
\hline 2 - Moderado x Severo & $22 / 28$ & 0,2 & S \\
\hline 3 - Baixo x Moderado & $21 / 28$ & 0,6 & S \\
\hline \multicolumn{4}{|l|}{ Pares de vinho - MP } \\
\hline 4 - Baixo x Severo & $25 / 28$ & 0,01 & $S^{* \star *}$ \\
\hline 5 - Moderado x Severo & $22 / 27$ & 0,1 & S \\
\hline 6 - Baixo x Moderado & $24 / 28$ & 0,01 & $S^{* * *}$ \\
\hline
\end{tabular}

Pares de vinhos

Baixo estresse hídrico

$\begin{array}{llll}7-\mathrm{HP} \times \mathrm{MP} & 21 / 27 & 0,3 & \mathrm{~S}\end{array}$

Estresse hídrico moderado
8 - HP x MP

Estresse hídrico severo

9 - HP x MP

$21 / 28$

0,6

$S$ 
número 8 evidencia que os dois sistemas de poda utilizados geraram vinhos que diferem significativamente no perfil sensorial, dentro do nível de estresse hídrico moderado.

0 detalhamento das diferenças significativas observadas nos pares de vinho da Tabela 4, foi feito através da análise descritiva quantitativa e está mostrado na Figura 3, notando-se que sob estresse hídrico severo (Figura 3A, B), os vinhos adquiriram características mais herbáceas e temperadas, independentemente do sistema de poda; por outro lado, os vinhos produzidos sob estresse hídrico baixo apresentam características de aroma frutado mais intenso. Quando se considera a variação de estresse hídrico baixo para moderado, observa-se maior complexidade de aroma (Figura $3 C$ ), para cujo caso, mantendo-se o mesmo sistema de poda (MP), em nível de estresse hídrico baixo, os vinhos elaborados tiveram perfil aromático com notas de frutas-secas e erva-doce mais intensas. A Figura 3D, porém, mostra que sob poda mecânica e estresse hídrico moderado os vinhos atingiram notas mais altas para frutado.

Portanto e apesar da análise química e os componentes do rendimento indicarem maior qualidade potencial da uva, em função do estresse hídrico e sistema de poda, para a safra de 2004 (Tabela 1), o perfil sensorial dos vinhos desta safra não mostra uma relação de maior intensidade aromáti-

\section{A.}

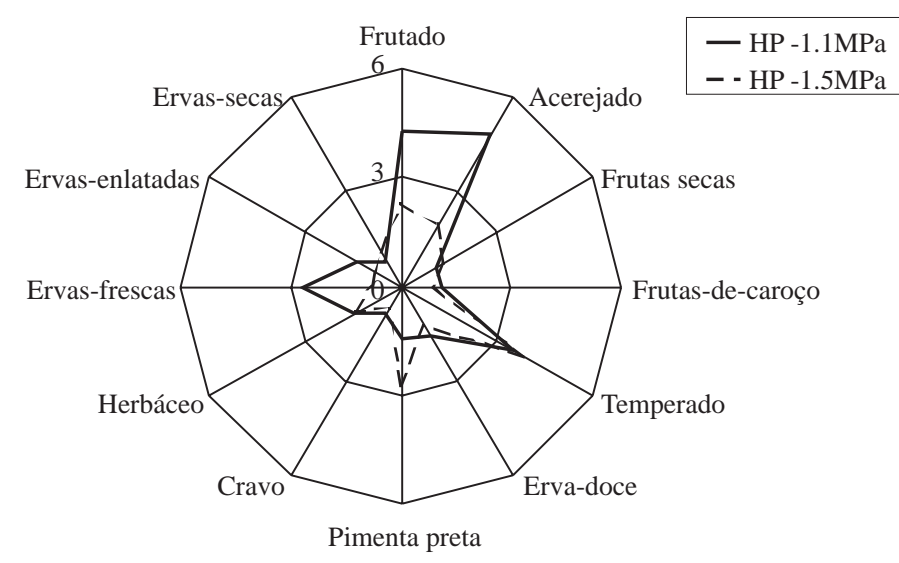

C.

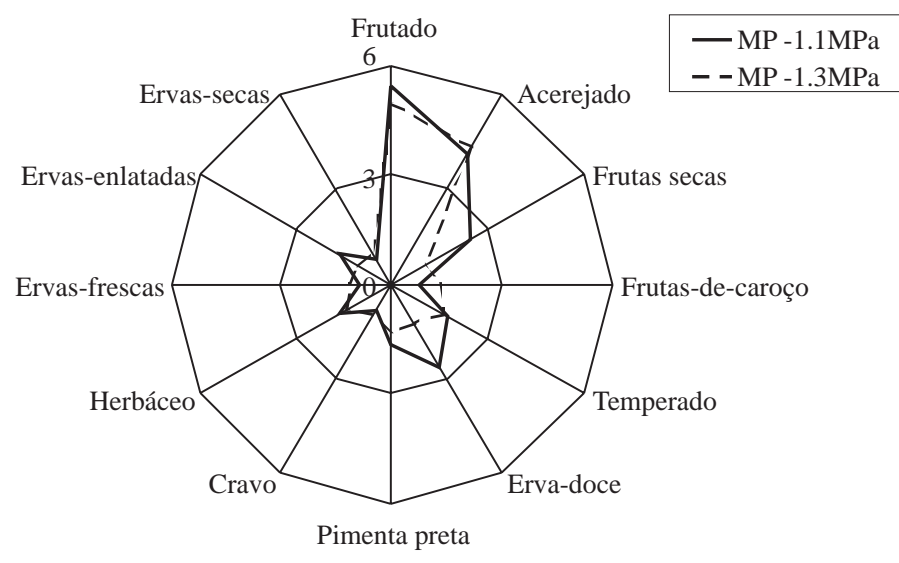

ca diretamente relacionada com o nível crescente de estresse hídrico transiente. Notadamente, dentro do mesmo sistema de poda o estresse hídrico de leve a moderado proporcionou vinhos com frutado mais intenso (Figura $3 \mathrm{~A}, \mathrm{~B}, \mathrm{C}$ ); esses mesmos resultados no vinho foram alcançados mantendo-se um nível de estresse hídrico moderado e se aplicando poda mecânica; por outro lado, os vinhos advindos de parcelas com estresse hídrico severo, tanto sob poda mecânica como manual, apresentam perfil aromático menos desenvolvido (Figura 3A, B); este último detal he contrasta com os resultados obtidos por Ojeda et al. (2004) para a mesma cultivar, em mesoclima mediterrâneo europeu, em que um estresse hídrico de médio a forte foi mais favorável para a produção de vinhos de qualidade. Depreende-se, deste fenômeno, que regimes microclimáticos diferentes renderão diferentes efeitos na qualidade do vinho quando se aplica restrição hídrica como estratégia de manejo.

\section{CONCLUSÕES}

1. A cultivar Syrah não apresentou tendência de queda sistemática de produtividade devido ao estresse intensificado ao longo dos anos.
B.

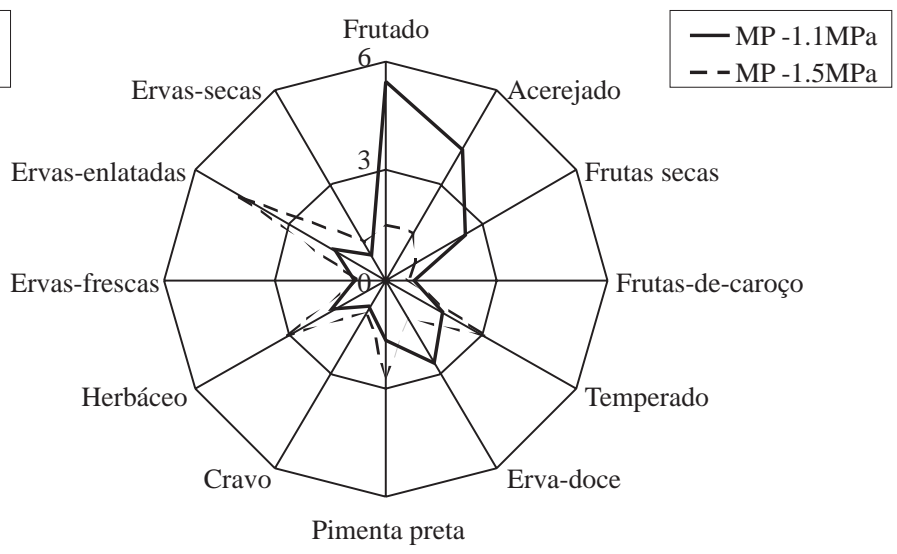

D.

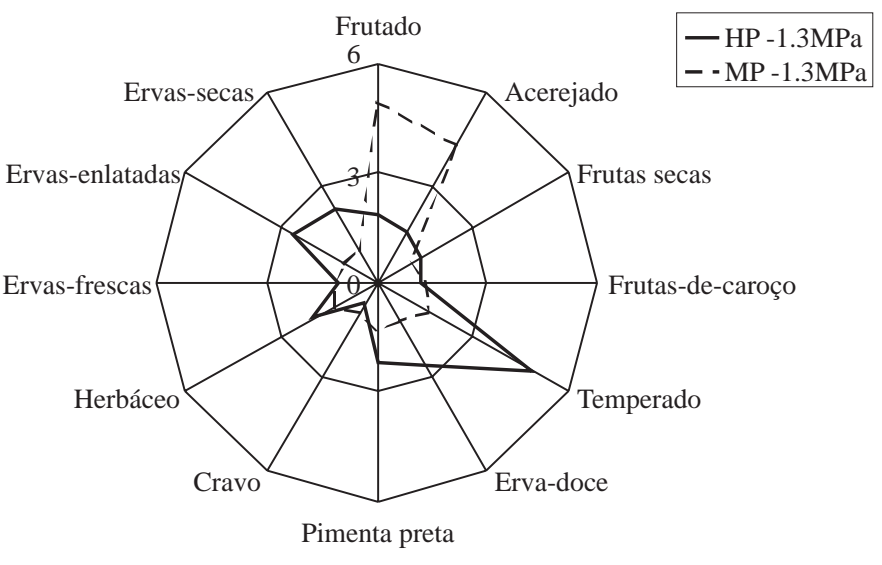

Figura 3. Perfil aromático dos pares de vinhos de Syrah, diferenciados estatisticamente no teste do triângulo, obtidos em experimentação com sistema de poda e estresse hídrico transiente, no Vale Central da Califórnia (EUA) 
2. Os sistemas de poda aplicados exerceram influência consistente sobre os componentes do rendimento ao longo de três safras.

3. Os níveis de estresse hídrico aplicados ao longo dos anos, diferenciaram os componentes do rendimento, embora de forma não consistente.

4. Os vinhos de 'Syrah' produzidos na faixa de estresse hídrico de leve a moderado e sob poda mecânica, tiveram perfil aromático mais desenvolvido.

\section{LITERATURA CITADA}

A morim, D. A.; Favero, A . C.; Regina, M . A . Produção extemporânea de videira, cultivar syrah, nas condições do Sul de M inas Gerais. Revista B rasileira de Fruticultura, v.27, n.2, p.327-331, 2005.

Bindi, M.; B ellesi, S.; Orlandini, S.; Fibbi, L.; M oriondo, M.; Sinclair, T. Influence of water deficit stress on leaf area development and transpiration of Sangiovese grapevines grown in pots. A merican Journal of Enology and Viticulture, v.56, p.68-72, 2005.

Bindon, K.; Dry, P.; Loveys, B. Influence of partial rootzone drying on the composition and accumulation of anthocyanins in grape berries (Vitis vinifera cv. Cabernet Sauvignon). A ustralian J ournal of Grape and Wine Research, v.4, n.2, p.91-103, 2008.

Bravdo, B.; Hepner, Y.; Loinger, C.; Cohen, S.; Tabacman, H. Effect of crop level en crop load on growth, yield, must and wine composition and quality of Cabernet Sauvignon. A merican Journal of Enology and Viticulture, v.36, p.125$131,1985$.

Deloire, A.; Hunter, J. J. Microclimat des grappes et maturation du raisin. Le Progrès A gricole et Viticole, v.122, n.7, p.125132, 2005.

Deloire A.; Lopez, F. Y.; Carbonneau, A. Réponses de la vigne et terroirs. Eléments pour une méthode d'étude. Le Progrès A gricole et Viticole, v.4, p.78-86, 2002.

Dokoozlian, N. K. Influence of row orientation and cluster exposure to sunlight on the microclimate and composition of cabernet sauvignon grapes. A nnual Progress Report, Parlier: UC Kearney A gricultural Center, 2001. 11p.

Dokoozlian, N. K.; K liewer, W. M. Influence of light on grape berry growth and composition varies during fruit development. Journal of A merican Society of Horticultural. Science, v.121, p.869-874, 1996.

Duncan, S. A pplication of sensory evaluation in wine making. In: Wine analysis and production. Zoecklein, B.; Fugelsang K.; N ury, F.; Gump B (ed.). M aryland: Chapman \& Hall, 1999. 43p.

Ferreyra R. E.; Sellés, V. J.; Peralta, A.; Val enzuela, J. Effect of water stress applied at different development periods of Cabernet Sauvignon grapevine on production and wine quality. A cta Horticulturae, v.646, p.27-33, 2004.

Freeman, B. M.; K liewer, W. M. Effect of irrigation, crop level and potassium fertilization on carignane vines. II Grape and Wine quality. A merican J ournal of Enology and Viticulture, v.34, p.197-207, 1983.
Freeman, B. M.; Lee, T. H.; Turkington, C. R. Interaction of irrigation and pruning level on grape and wine quality of shiraz vines. A merican Journal of Enology and Viticulture, v.31, p.124-135, 1980.

Fugelsang, K. C. Wine microbiology. New York: Chapman \& Hall, 1996. 150p.

Galpin, V. C. A comparison of legislation about winemaking additives and process. South A frican Computer Journal, v.26, p.13-21, 2006.

Ginestar, C.; Eastham, J.; Gray, S.; Iland, P. U se of sap-flow sensors to schedule vineyard irrigation. II. Effects of postveraison water deficits on composition of Shiraz grape. A merican Journal of Enology and Viticulture, v.49, n.4, p.421-428, 1998.

Girona, J.; M ata, M.; del Campo, J.; A rbonés, J.; Bartra, E.; M arsal, J. The use of midday leaf water potencial for scheduling déficit irrigation. Irrigation Science, v.24, p.115-127, 2006.

Gurovich, L.; Vergara, M. Riego deficitario controlado: la clave para la expresión del terroir de vinos premium. In: Seminario Internacional de M anejo de Riego y Suelo en Vides para Vino y M esa, 1, 2005, Santiago. Proceedings... Santiago: Instituto de Investigaciones A gropecuarias, 2005. 30p.

Hardie, W. J.; Considine, J. A. Response of grapes to water-deficit stress in particular stages of development. A merican Journal of Enology and Viticulture, v.27, p.55-61, 1976.

Hsiao, T. C. Plant responses to water stress. A nnual. Review of Plant Physiology, v.24, p.519-570, 1973.

Iland, P.; Bruer, N.; Edwards, G.; Weeks, S.; Wilkes, E. Chemical analysis of grapes and wine: techniques and concepts. A delaide: Tony Kitchener Printings, 2004. 110p.

Jackson, D. I.; Lombard, P. B. Environmental and management practices affecting grape composition and wine quality: A review. A merican Journal of Enology and Viticulture, v.44, p.409-430, 1993.

Jensen, J. S.; Blachez, B.; Egebo, M.; Meyer, A. S.; Rapid extraction of polyphenols from red grapes. A merican J ournal of Enology and Viticulture, v.587, p.451-461, 2007.

Kasimatis, A. N. Differential cropping levels of zinfandel wines - a progress report on some effects on vine growth, fruit composition and wine quality. In: International Symposium on Quality of Vintage, 10, 1977, CapeTown. Proceedings... CapeTown: O enological and Viticulture Research Institute, 1977. p.189-194.

Keller, M.; Smithyman, R. P.; M ills, L. J. Interactive effects of deficit irrigation and crop load on cabernet sauvignon in an arid climate. A merican Journal of Enology and Viticulture, v.59, p.221-234, 2008.

Kupina, S. A.; Shrikhande, A. J. Evaluation of a fourier transform infrared instrument for rapid quality-control wine analyses. A merican Journal of Enology and Viticulture, v.54, n.2, p.131134, 2003.

Lawless, H.; Heymann, H. Sensory eval uation of food-principles and practices. New York: Chapman \& Hall, 1998. 827p.

Noble, A. C.; A rnold, J.; B uechsenstein, A .; L each, E. J.; Schmidt, J. O.; Stekrn, P. M. M odification of a standardized system of wine aroma terminology. A merican Journal of Enology and Viticulture, v.38, n.1, p.143-146, 1987. 
Ojeda, H.; Deloire, A.; Wang, Z.; Carbonneau, A. Determinación del estado hídrico de la vid. Efectos morfológicos y fisiológicos de la restriçión hídrica em vides. Viticultura e Enologia Profissional, v.90, p.27-43, 2004.

Poni, S.; Lakso, A. N.; Turner, J. R.; Melious, R. E. Interactions of crop level and late season water stress on growth and physiology of field-grown Concord grapevines. A merican Journal of Enology and Viticulture, v.45, p.252-257, 1994.

Rizzon, L. A .; M iele, A. Avaliação da cultivar Cabernet Sauvignon para elaboração de vinho tinto. Ciência e Tecnologia de A limentos, v.22, n.2, p.192-198, 2002.

Soar, C. J.; L oveys, B. The effect of changing patterns in soilmoisture availability on grapevine root distribution, and viticultural implications for converting full-cover irrigation into a point-source irrigation system. A ustralian J ournal of Grape and Wine Research, v.13, n.1, p. 2-13, 2007.

Souza, C. M ; Regina, M. A.; Pereira, G. E.; Freitas, G. F. Indicação de cultivares de videira para o Sul de M inas Gerais. In: Regina, M.A. (ed.) . Viticultura e enologia: A tualizando conceitos. Caldas: EPAM IG-FECD, 2002. p.277-286.
Tarara, J. M.; L ee, J.; Spayd, S. E.; Scagel, C. Berry temperature and solar radiation alter acylation, proportion, and concentration of anthocyanin in merlot grapes. A merican Journal of Enology and Viticulture, v.59, p.235-247, 2008.

Teixeira, A. H. C.; Souza, R. A.; Ribeiro, P. H. B.; Reis, V. C. S.; Santos, M . G. L. A ptidão agroclimática da cultura da videira no Estado da Bahia. Revista Brasileira de Engenharia A grícola e A mbiental, v.6, n.1, p. 107-111, 2002.

Williams, L. E.; M athews, M. A. Grapevines. In: Irrigation of agricultural crops. Stewart, B. A.; Nielsen, D. R. (eds.), A merican Society of A gronomy, v.30, p.1019-1055, 1990.

Winkler, A. J.; Cook, J. A.; Kliewer, W. M.; Lider, L. A. General viticulture. Berkeley: University of California Press, 1974. 130p.

Zoecklein, B.; Fugelsang, K.; N ury, F.; Gump, B. Wine analyses and production. New York: Chapman \& Hall, 1999. 390p.

Zyl, J. L.; Weber, H. W. I Irigation of Chenin blanc In the Stellenbosch area within the framework of climate-soil-water plant continuum. In: International Symposium on quality of vintage, 10, 1977, CapeTown. Proceedings... CapeTown: Oenological and Viticulture Research Institute, 1977. p.150-156. 